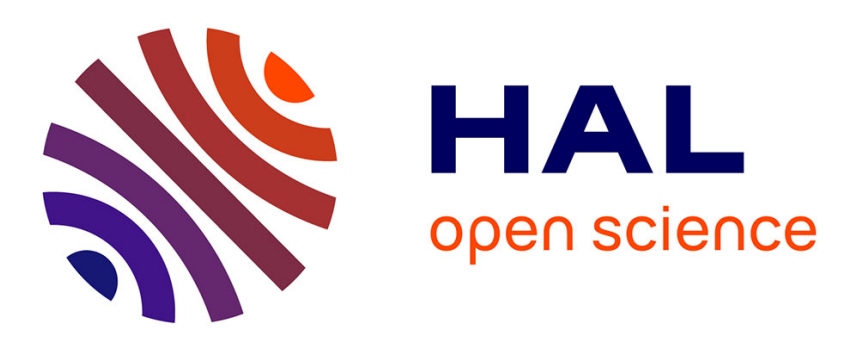

\title{
Empirical SURE-guided microscopy super-resolution image reconstruction from confocal multi-array detectors
}

\author{
Sylvain Prigent, Stéphanie Dutertre, Charles Kervrann
}

\section{To cite this version:}

Sylvain Prigent, Stéphanie Dutertre, Charles Kervrann. Empirical SURE-guided microscopy superresolution image reconstruction from confocal multi-array detectors. ICASSP 2020 - 2020 IEEE International Conference on Acoustics, Speech and Signal Processing, May 2020, Barcelona, Spain. pp.1-5. hal-03087023

\section{HAL Id: hal-03087023 \\ https://hal.inria.fr/hal-03087023}

Submitted on 23 Dec 2020

HAL is a multi-disciplinary open access archive for the deposit and dissemination of scientific research documents, whether they are published or not. The documents may come from teaching and research institutions in France or abroad, or from public or private research centers.
L'archive ouverte pluridisciplinaire HAL, est destinée au dépôt et à la diffusion de documents scientifiques de niveau recherche, publiés ou non, émanant des établissements d'enseignement et de recherche français ou étrangers, des laboratoires publics ou privés. 


\title{
EMPIRICAL SURE-GUIDED MICROSCOPY SUPER-RESOLUTION IMAGE RECONSTRUCTION FROM CONFOCAL MULTI-ARRAY DETECTORS
}

\author{
Sylvain Prigent ${ }^{1}$, Stéphanie Dutertre ${ }^{2}$, Charles Kervrann ${ }^{3}$ \\ ${ }^{1}$ France-BioImaging, France \\ ${ }^{2}$ University of Rennes 1, UMS Biosit, MRic, France \\ ${ }^{3}$ Inria Centre Rennes-Bretagne Atlantique, France
}

\begin{abstract}
The new generation of confocal microscopes are equipped with an array detector that generates an array of images corresponding to a multiview of the same sample. Several computational methods have been proposed to reconstruct a single super-resolution image from a stack of images associated to detectors. Each method has its pros and cons depending on the targeted application. In this paper, we review the most commonly used reconstruction methods and propose a $S U R E$ approach to automatically estimate parameters and improve reconstruction. Methods described in this paper are available in an open source software.
\end{abstract}

Index Terms - Super-resolution, image reconstruction, confocal imaging, SURE, estimation

\section{INTRODUCTION}

Recent confocal microscopes replace the single point detector with an array detector that allows to take multiple views of the same sample [1]. Such microscope raw data is an array of images, one image per detector. The array of images is then processed to build a single image with higher signalto-noise ratio and higher resolution than a classical confocal microscope image.

Several methods have been recently proposed to reconstruct this single high resolution image. In [1], the authors combine array registration and Wiener deconvolution. In [2], a method (IFED) creates a high resolution image by subtracting the outer detectors of the array to the inner detectors (see Fig. 1). In [3], the authors modify the IFED method to improve the resolution with a method called ISFED. Each method is based on a pipeline involving image processing tasks, including array image registration, array image linear combination, and optional deconvolution. Each of these methods have their pros and cons, and one may be more suitable than another, depending on the target application. For instance, for intensity quantification, it is recommended to choose a method using only array image linear combination to reduce intensity bias, whereas one could prefer to apply a deconvolution step to improve resolution of structures.



Fig. 1. Detector arrangement. The 32 detectors are arranged around the central detector. The numbers are the detector indexes, the dark blue circle highlight the group of $N=7$ central detectors and the light blue circle highlight the group of $N=19$ central detectors. Detectors inside circles are called inner detectors whereas detectors outside circles are called outer detectors.

In the next section, we briefly describe the current reconstruction methods. In Section 3, we propose a SURE-guided (Stein's unbiased risk estimation) estimation method and show on real data that the proposed reconstruction method achieves a resolution close to $100 \mathrm{~nm}$ without any deconvolution method. Unlike [2, 3], we show that the parameter depends on the image contents and signal intensities. In section 4 , we use a method named ISM deconvolution (ISM-D) as a reference, since it is established that provides higher resolution images [1]. Nevertheless, it is computationally costly and can introduce artifacts.

\section{RECONSTRUCTION METHOD}

In summary, an array detector is made of 32 Gallium arsenide phosphide (GaAsP) detectors elements arranged in a compound eye fashion (see Fig. 1). The central element is in the optical axis, and the other elements are grouped around. The full detector is 1.25 Airy units (AU) large and thus a detector is approximately 0.2 AU large. One AU is the diameter of the airy disk obtained by imaging a point.

Given a set of input images (or detector array) $I_{i}, i=$ $1 \cdots N$, we describe five methods to reconstruct a single image: pseudo-confocal (PC), ISM, IFED, ISFED and ISM deconvolution (ISM-D).

The pseudo-confocal reconstruction technique sums the 
detectors as follows:

$$
I_{P C}(x)=\sum_{i=1}^{N} I_{i}(x)
$$

where $I_{i}(x)$ is the intensity at pixel $x \in \Omega \subset \mathbb{R}^{2}$ of the $2 \mathrm{D}$ image from the detector at index $i$ where $N \in\{7,19,32\}$ is the number of detectors. If $N$ is small (eg. $N=7$ ) we get more resolution but less signal. This reconstruction methods gives an image equivalent to a confocal microscope with a high signal-to-noise-ratio.

The ISM reconstruction technique [1] consists in adding the co-registered images $\left\{\tilde{I}_{i}\right\}_{i=1}^{32}$ from the 32 detectors:

$$
I_{I S M}(x)=\sum_{i=1}^{32} \tilde{I}_{i}(x)
$$

where $\tilde{I}_{i}(x)$ is the intensity at pixel $x$ of the image from the detector at index $i$, co-registered on the central detector. This method gives an image with a theoretical resolution of 0.2 AU.

The IFED reconstruction has been proposed to improve resolution by subtracting the outer detectors ring to the inner detectors [2] (see Fig. 1):

$$
I_{I F E D}(x)=\sum_{i=1}^{N} I_{i}(x)-\epsilon \sum_{i=N+1}^{32} I_{i}(x),
$$

where $\epsilon \in[0,1]$ is a subtraction coefficient to be hand-tuned. In [2], the authors showed that the IFED method achieves a higher resolution than the classical ISM reconstruction method.

In [3], the authors defined ISFED, which is a modification of IFED, to get more spatial resolution:

$$
I_{I S F E D}(x)=\sum_{i=1}^{32} \tilde{I}_{i}(x)-\epsilon \sum_{i=1}^{32} I_{i}(x), \epsilon>0 .
$$

The aforementioned methods allow to improve resolution without any deconvolution process. IFED ans ISFED need the adjustment of a parameter $\epsilon$ to get satisfying results. In what follows, we propose a method to automatically estimate this parameter.

\section{EMPIRICAL SURE FOR IFED AND ISFED PARAMETER ESTIMATION}

IFED and ISFED methods are based on the subtraction of two sets of detectors. To generalize the idea $[2,3]$, we define the reconstructed image $\hat{u}(x)$ at pixel $x \in \Omega$ as:

$$
\begin{aligned}
\hat{u}(x) & =K_{h} *\left(\sum_{i=M_{1}}^{M_{2}} I_{i}(x)-\epsilon \sum_{i=N_{1}}^{N_{2}} I_{i}(x)\right) \\
& =a_{I}(x)-\epsilon b_{I}(x)
\end{aligned}
$$

where $h$ is the bandwidth of the convolution filter $K$ and $*$ is the convolution operator. In what follows, $a_{I}(x)=K_{h} *$ $\sum_{i=M_{1}}^{M_{2}} I_{i}(x)$ is the sum of the detectors with indexes between $M_{1}$ and $M_{2}$, and $b_{I}(x)=K_{h} * \sum_{i=N_{1}}^{N_{2}} I_{i}(x)$ is the sum of the detectors with indexes between $N_{1}$ and $N_{2}$, and $\epsilon>0$ is a constant. In the case of IFED, $M_{1}=1, M_{2}=N$, $N_{1}=N+1$ and $N_{2}=32$, and in the case of ISFED, $M_{1}=1$, $M_{2}=32, N_{1}=1$ and $N_{2}=32$. The bandwidth $h$ is chosen very small to slightly reduce noise while preserving high frequencies.

Let us consider a neighborhood $\Omega(x) \subset \Omega$ centered at point $x$ and let us denote $n=|\Omega(x)|$ the number of pixels in $\Omega(x)$. We propose to estimate $\epsilon$ by using the local Stein's unbiased risk estimate $(S U R E)[4,5]$ of $\hat{u}(x)$ with respect to the reference central detector $I_{1}$ and defined as:

$$
S U R E(x)=\frac{1}{n} \sum_{y \in \Omega(x)}(I(y)-\hat{u}(y))^{2}-\sigma^{2}+\frac{2 \sigma^{2}}{n} \operatorname{div}_{I}(\hat{u}),
$$

where $I(y)=I_{1}(y)$ is the image of the central detector, $\sigma^{2}$ is the variance of the assumed white Gaussian noise and

$$
\operatorname{div}_{I}(\hat{u})=\sum_{y \in \Omega(x)} \sum_{i=1}^{N} \frac{\partial \hat{u}(y)}{\partial I_{i}(y)}
$$

Since $a_{I}(y)$ and $b_{I}(y)$ are constant given $I_{i}(y)$ it follows that:

$$
\operatorname{div}_{I}(\hat{u})=\sum_{y \in \Omega(x)}\left(M_{2}-M_{1}\right)-\epsilon\left(N_{2}-N_{1}\right) .
$$

The $S U R E$ risk is then written as:

$$
\begin{aligned}
S U R E(x) & =\frac{1}{n} \sum_{y \in \Omega(x)}\left(I(y)-a_{I}(y)+\epsilon b_{I}(y)\right)^{2} \\
& -\sigma^{2}+2 \sigma^{2}\left(M_{2}-M_{1}-\epsilon\left(N_{2}-N_{1}\right)\right) .
\end{aligned}
$$

We define the empirical $S U R E$ as the sum of local $S U R E$ as:

$$
S U R E=\sum_{x \in \Omega} S U R E(x)
$$

which is an unbiased estimator of the Mean Square Error (MSE), that is:

$$
\begin{aligned}
\mathbb{E}[S U R E] & =\sum_{x \in \Omega} \mathbb{E}[\operatorname{SURE}(x)] \\
& =\sum_{x \in \Omega} \mathbb{E}\left[(\hat{u}(x)-u(x))^{2}\right]=\mathbb{E}\left[\|\hat{u}-u\|^{2}\right]
\end{aligned}
$$

It is worth noting that, when the neighborhood $\Omega(x)$ is reduced to a single pixel $x$, the empirical SURE is nothing else that the conventional $S U R E$ on domain $\Omega$. It follows that solving $\frac{\partial S U R E(x)}{\partial \epsilon}=0$ gives the following closed-form solution for $\epsilon$ : 


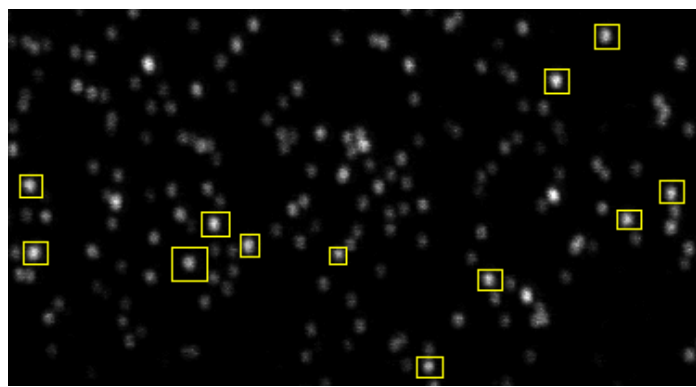

(a)

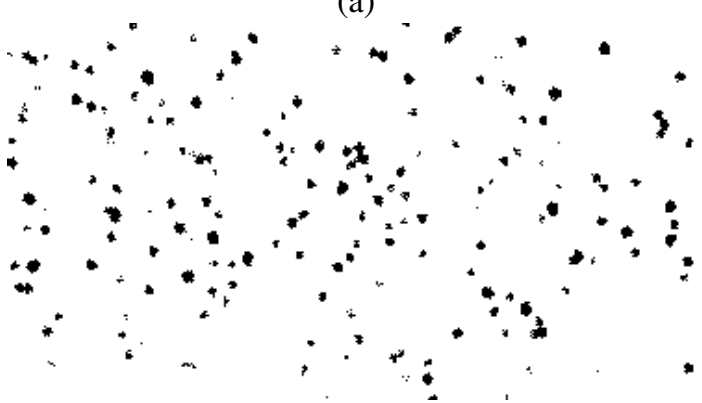

(b)

Fig. 2. Image depicting beads of $100 \mathrm{~nm}$ diameter used for resolution measurement. (a) $I_{p c}(x)$ image with squares showing regions used to select 12 beads for measurement. (b) Binary image of Otsu threshold on $I_{1}(x)$.

$\epsilon=\frac{\sum_{x \in \Omega}\left[\left(N_{2}-N_{1}\right) \sigma^{2}-n^{-1} \sum_{y \in \Omega(x)}\left(I(y)-a_{I}(y)\right) b_{I}(y)\right]}{\sum_{x \in \Omega} n^{-1} \sum_{y \in \Omega(x)} b_{I}^{2}(y)}$.

Microscopy image contents is mainly background (see Fig. 2,4 and 3). In practice, we then restrict $\Omega^{\prime}$ to the area of the image where signal is significant given a threshold $\tau: \Omega^{\prime} \subset$ $\Omega=\left\{x: I_{1}(x)>\tau\right\}$. Hence, $\Omega^{\prime}$ is substituted to $\Omega$ in (12).

\section{EXPERIMENTAL RESULTS}

We evaluated the proposed $\epsilon$ estimation method on an image of $100 \mathrm{~nm}$ diameter beads (see Fig. 2(a)). In all experiments, the set of input images are slightly filtered by applying a Gaussian filter with a bandwidth $h=0.5$. First, we evaluated the sensitivity of the $S U R E$ estimation depending on the size of $\Omega(x)$ and $\tau$. The bead intensity range is $[0,130]$ and the majority of the beads have intensities below 80 . Thus, for $\tau$, we arbitrary chose 8 threshold values in the range $[0,70]$, and for $\Omega(x)$ we chose square neighborhoods from $1 \times 1$ pixel to $15 \times 15$ pixels. The estimated values for $I F E D$ are reported in Table 1. The first column is the conventional SURE $(1 \times 1$ pixel). One can notice that the conventional $S U R E$ estimation of $\epsilon$ is very sensitive to $\tau$ whereas for $\Omega(x)$ larger than a $9 \times 9$ square, the empirical $S U R E$ estimation of $\epsilon$ is very stable. Calculations made for the ISFED methods gave the same conclusions. We then conclude that the empirical $S U R E$ method is more robust and the size of $\Omega(x)$ and $\tau$ do not require accurate adjustment. In the following, we estimate

\begin{tabular}{|c|c|c|c|c|c|c|c|c|}
\hline$\tau_{\tau} \quad \Omega(x)$ & $1 \times 1$ & $3 \times 3$ & $5 \times 5$ & $7 \times 7$ & $9 \times 9$ & $11 \times 11$ & $13 \times 13$ & $15 \times 15$ \\
\hline 0 & 0.76 & 0.76 & 0.76 & 0.76 & 0.76 & 0.76 & 0.76 & 0.76 \\
\hline 5 & 0.85 & 0.87 & 0.87 & 0.85 & 0.82 & 0.79 & 0.77 & 0.76 \\
\hline 10 & 0.92 & 0.92 & 0.90 & 0.86 & 0.82 & 0.79 & 0.76 & 0.75 \\
\hline 15 & 0.97 & 0.96 & 0.92 & 0.87 & 0.82 & 0.78 & 0.76 & 0.75 \\
\hline 25 & 1.06 & 1.02 & 0.95 & 0.88 & 0.82 & 0.78 & 0.77 & 0.76 \\
\hline 40 & 1.16 & 1.09 & 0.98 & 0.89 & 0.82 & 0.79 & 0.77 & 0.76 \\
\hline 50 & 1.21 & 1.12 & 0.99 & 0.89 & 0.82 & 0.79 & 0.78 & 0.77 \\
\hline 70 & 1.30 & 1.18 & 1.01 & 0.89 & 0.82 & 0.79 & 0.77 & 0.77 \\
\hline Avera & 1.03 & 0.99 & 0,92 & 0,86 & 0,81 & 0,78 & 0,77 & 0,76 \\
\hline
\end{tabular}

Table 1. $\epsilon$ values depending on $\tau$ and $\Omega(x)$. $\tau$ values are indicated in pixel intensity of $I_{1}(x) \in[0,130]$. The neighborhood $\Omega(x)$ are squares patches of size $\mathrm{N}$.

\begin{tabular}{lc} 
Method & FWMH \\
\hline PC & $189 \mathrm{~nm}$ \\
ISM & $171 \mathrm{~nm}$ \\
IFED $\epsilon=0.3$ & $171 \mathrm{~nm}$ \\
ISFED $\epsilon=0.3$ & $159 \mathrm{~nm}$ \\
SURE-guided IFED & $137 \mathrm{~nm}$ \\
SURE-guided ISFED & $124 \mathrm{~nm}$ \\
ISM-D & $99 \mathrm{~nm}$
\end{tabular}

Table 2. Resolution measured for each method using 12 beads FWMH from the image of Figure 2.

$\tau$ by using the Otsu estimation method [6] and set $|\Omega(x)|$ to $11 \times 11$. With these settings, we obtained $\epsilon=0.78$ (IFED) and $\epsilon=1.1$ (ISFED).

We then performed the reconstruction with all the methods presented in Section 2 and evaluated the resolution by measuring the full width at half maximum (FWHM) intensity on 12 beads highlighted by squares in Fig. 2 . Results are summarized in Table 2 and sorted by increasing resolution. We can see that the SURE-guided method gives the closest resolution to the expected $100 \mathrm{~nm}$ resolution, and only the $I S M-D$ (Wiener) allows to recover the $100 \mathrm{~nm}$ resolution.

We tested the proposed method on images of intestinal microvilli from an adult c.elegans worm expressing the EMR1 fusion GFP protein (see Fig. 4). We chose this specimen since it depicts parallel line like structures of $100 \mathrm{~nm}$ large that ease the visualization of the resolution gain. In our experiments, we applied the $I S M-D$ method to asses reconstruction performances. We used 2 implementations of ISM-D, one using Wiener deconvolution [1] (ISM-DW) and the other using the recent Sparse Deconvolution algorithm (ISM-DS) [7] which is adapted to fluorescence microscopy images. For the IFED method, our SURE-guided method provided $\epsilon=0.38$. The estimated value is close to $\epsilon=0.30$ that was experimentally proposed in [3]. For the ISFED we obtained $\epsilon=0.95$ which is different from the experimentally value $\epsilon=0.30$ recommended in [3]. In Fig. 4 we can see that for both IFED and ISFED the SURE-guided estimation gives a better visualization of the line structures than the default $\epsilon=0.3$ value. Furthermore, we noticed that the ISFED method gives the closest reconstruction result to the $I S M-D$ references even though the 


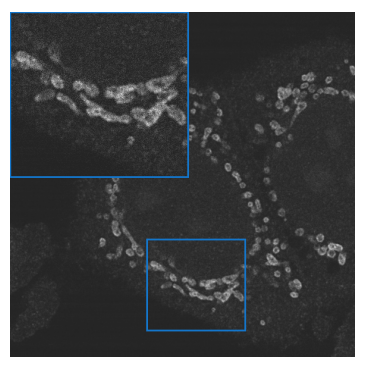

SURE-guided IFED

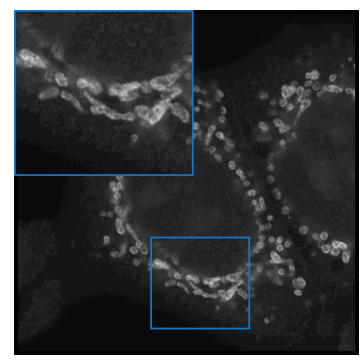

ISM-DW

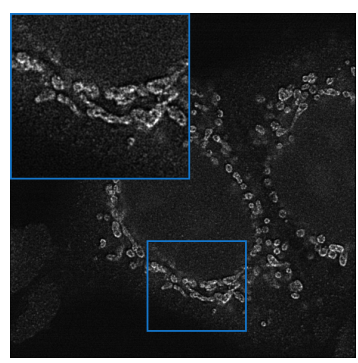

SURE-guided ISFED

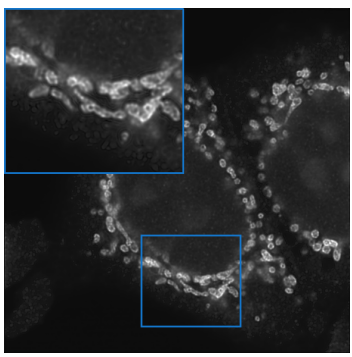

ISM-DS
Fig. 3. Results obtained on MCF7 cells expressing mito-GFP (labelling mitochondria) sample with all the tested methods.

signal to noise ratio is lower. In fact, when we examine at the intensity profiles (see Fig. 4), each peak corresponds to an intestinal microvilli. The profile estimated by the SURE-guided $I S F E D$ is the closest to the $I S M-D$ profile. As a conclusion for this c.elegans specimen, the best reconstruction without deconvolution is the SURE-guided ISFED.

We also tested the proposed method on images of mitochondria of MCF7 cells expressing mito-GFP. Mitochondria structures are visible with a resolution below $200 \mathrm{~nm}$. For this specimen, $\epsilon=0.33$ (IFED) and $\epsilon=0.96$ (ISFED). Again, the SURE-guided ISFED reconstruction gives the resolution the closest $I S M-D$ references (see Fig. 3).

\section{CONCLUSION}

In this paper, we reviewed five methods to generate superresolution images from array detector confocal microscope, and proposed a SURE-guided method to estimate the parameters controlling the IFED and ISFED methods. We showed on real data that the proposed approach allows to get the best resolution without parameter tuning. Thus, the only user input is the choice of the method depending on the target application. In our experiments, we applied a linear Gaussian filter. Nevertheless, it is possible to first denoise the input images with more sophisticated algorithms such as ND-SAFIR (https: //team.inria.fr/serpico/software/) [8]. The work presented in this paper has been implemented in an open source software available at https: //team.inria.fr/ serpico/airyscanj/.

\section{ACKNOWLEDGMENT}

The authors would like to thank Aurélien Bidaud-Meynard (IGDR) et Giulia Bertolin (IGDR) for providing the biolog-

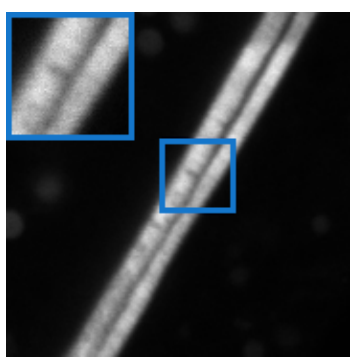

Pseudo-confocal

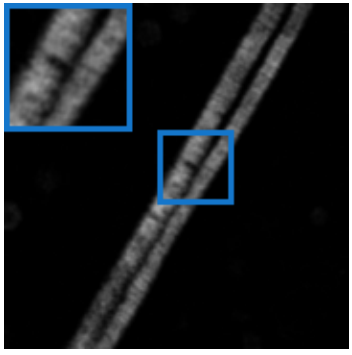

SURE-guided IFED

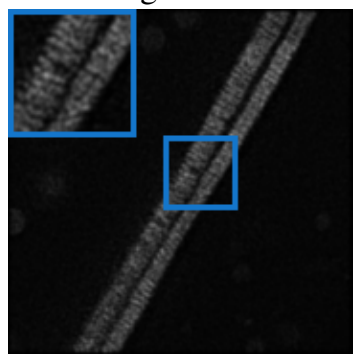

SURE-guided ISFED

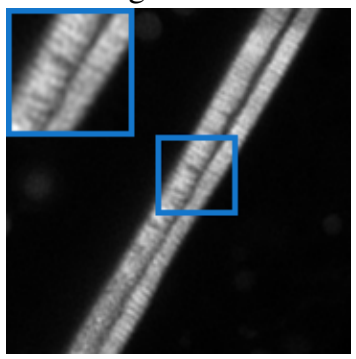

ISM-DW



ISM

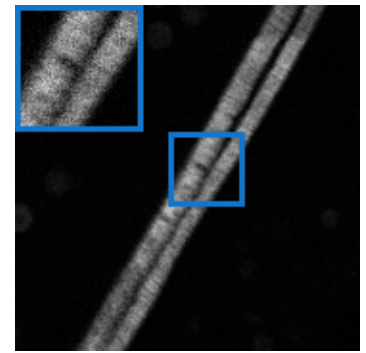

IFED $\epsilon=0.3$

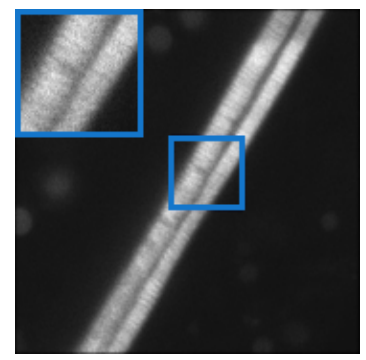

ISFED $\epsilon=0.3$

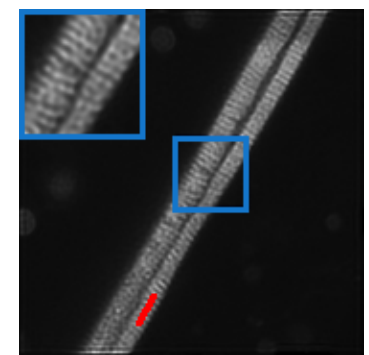

ISM-DS



Intensity profiles

Fig. 4. Results obtained on a c.elegans sample and intensity profiles for all the tested methods. The profile line is shown with a red line on the $I S M-D S$ image.

ical samples. This work was also supported by the French National Research Agency (France-BioImaging infrastructureANR10-INBS-04) 


\section{REFERENCES}

[1] Klaus Weisshart, "The basic principle of airyscanning," ZEISS Technology Note, 2014.

[2] Baoliang Ge, Yifan Wang, Yujia Huang, Cuifang Kuang, Yue Fang, Peng Xiu, Zihao Rong, and Xu Liu, "Threedimensional resolution and contrast-enhanced confocal microscopy with array detection," Opt. Lett., vol. 41, no. 9, pp. 2013-2016, May 2016.

[3] Yicheng Li, S. LIU, Diyi Liu, Sun Shiyi, C. KUANG, Z. DING, and X. LIU, "Image scanning fluorescence emission difference microscopy based on a detector array: Isfed based on a detector array," Journal of Microscopy, vol. 266, 022017.

[4] T. Qiu, A. Wang, N. Yu, and A. Song, "Llsure: Local linear sure-based edge-preserving image filtering," IEEE Transactions on Image Processing, vol. 22, no. 1, pp. 8090, Jan 2013.

[5] F. Luisier, T. Blu, and M. Unser, "SURE-LET for orthonormal wavelet-domain video denoising," IEEE Transactions on Circuits and Systems for Video Technology, vol. 20, no. 6, pp. 913-919, June 2010.

[6] N. Otsu, "A threshold selection method from gray-level histograms," IEEE Transactions on Systems, Man, and Cybernetics, vol. 9, no. 1, pp. 62-66, Jan 1979.

[7] Hoai-Nam Nguyen, Vincent Paveau, Cyril Cauchois, and Charles Kervrann, "Generalized sparse variation regularization for large fluorescence image deconvolution," Inria Research Report, 2017.

[8] J. Boulanger, C. Kervrann, P. Bouthemy, P. Elbau, J. Sibarita, and J. Salamero, "Patch-based nonlocal functional for denoising fluorescence microscopy image sequences," IEEE Transactions on Medical Imaging, vol. 29, no. 2, pp. 442-454, Feb 2010. 